\title{
Interface control in organic heterojunction photovoltaic cells by phase separation processes
}

\author{
Jakob Heier*, Fernando A. Castro, Frank Nüesch, Roland Hany \\ Empa, Swiss Federal Laboratories for Materials Testing and Research, Laboratory for Functional \\ Polymers, Ueberlandstr. 129, CH-8600 Duebendorf, Switzerland.
}

\begin{abstract}
Significant progress is being made in the photovoltaic energy conversion using organic semiconducting materials. One of the focuses of attention is the nanoscale morphology of the donor-acceptor mixture, to ensure efficient charge generation and loss-free charge transport at the same time. Using small molecule and polymer blend systems, recent efforts highlight the problems to ensure an optimized relationship between molecular structure, morphology and device properties. Here, we present two examples using a host/guest mixture approach for the controlled, sequential design of bilayer organic solar cell architectures that consist of a large interface area with connecting paths to the respective electrodes at the same time. In the first example, we employed polymer demixing during spin coating to produce a rough interface: surface directed spinodal decomposition leads to a 2-dimensional spinodal pattern with submicrometer features at the polymer-polymer interface. The second system consists of a solution of a blend of small molecules, where phase separation into a bilayer during spin coating is followed by dewetting. For both cases, the guest can be removed using a selective solvent after the phase separation process, and the rough host surface can be covered with a second active, semiconducting component. We explain the potential merits of the resulting interdigitated bilayer films, and explore to which extent polymer-polymer and surface interactions can be employed to create surface features in the nanometer range.
\end{abstract}

Keywords: organic solar cell, heterojunction, phase separation, liquid-liquid dewetting

\section{INTRODUCTION}

In recent years, tremendous efforts have been made to develop solar cells from organic materials. Organic semiconductors have the potential to absorb light and to transport electric charges. Together with their availability and their ease of processing, these materials are ideal candidates to serve as photovoltaic materials of the future ${ }^{1,2,3}$. Converting light into electrical current in organic materials is a four stage process: (i) light absorption leads to the formation of a bound electron-hole pair (exciton), (ii) the exciton diffuses to a region where it is (iii) separated into free charge carriers, and (iv) the charges are transported to the corresponding electrodes. In organic devices step (iii) can be achieved at a heterogeneous interface. This can be a metal-organic interface such as in organic Schottky devices or a fully organic heterointerface. For the latter, the simplest device consists of a bilayer, where an electron donor and an acceptor material are stacked together with a planar interface ${ }^{4}$. The excitons can be dissociated at the interface between the materials and the opposite charges are transported selectively to the corresponding electrode. However, a bottleneck for the realization of an efficient planar bilayer organic solar cell is the small diffusion length $(10-20 \mathrm{~nm})$ of the primary photo-excitations. Thus, only excitons generated in the direct vicinity of the interface contribute to charge generation. Bulk heterojunction cells based on the same two-component concept as the bilayer device, but with a largely increased interfacial area, are fabricated by applying a mixture of a donor and acceptor material directly. In favorable cases, these systems will develop phase separated domains of sizes comparable to the exciton diffusion length ${ }^{5,6}$, however, charge collection requires a bicontinuous interpenetrating network which proves to be demanding in fabrication.

A device architecture which is conceptually situated between the bilayer and the bulk heterojunction device is the socalled diffuse bilayer heterojunction device. This geometry aims to combine the uninterrupted pathways to the electrodes of the pure bilayer with the enlarged acceptor-donor interface of the bulk heterojunction device. Different approaches have been reported on how to achieve such a diffuse interface: one method is to spin coat the second layer from a solvent

\footnotetext{
* Jakob.Heier@empa.ch; phone +41 44823 4356; fax +41 448234012
}

Organic Photovoltaics VIII, edited by Zakya H. Kafafi, Paul A. Lane

Proc. of SPIE Vol. 6656, 66560P, (2007) -0277-786X/07/\$18 $\cdot$ doi: 10.1117/12.732432 
that partially dissolves the underlying polymer ${ }^{7}$, alternatively, annealing of the completed bilayer device will result in interdiffusion, also resulting in an intermixed interfacial region ${ }^{8}$.

We recently proposed another, more controlled method to enlarge the interfacial area, ${ }^{9,10}$. Thereby, an immiscible holeconducting polymer / guest polymer mixture was spin coated in a first step to produce a vertically segregated film with a highly folded interface. Subsequently, the guest material was removed using a selective solvent, and the remaining structured layer was covered with an electron-accepting material. Using a donor poly(p-phenylenevinylene) derivative, polystyrene as guest and the $\mathrm{C}_{60}$ fullerene as electron acceptor, solar cell efficiencies increased by a factor of 3 compared to the planar bilayer configuration, supporting the concept ${ }^{9}$. With the guest-polymer approach, we bypass the restrictions imposed by working directly with a hole-conducting/electron-conducting polymer blend. First of all, not every combination of active materials has to be optimized from scratch, secondly, the generally large interaction parameters between any two active components do not favor the formation of nanoscale structures at all.

When spin coating thin films out of a blend solution, we have to distinguish between several structure-directing mechanisms. Intermolecular interactions can lead to blend phase separation. While phase separation in bulk samples leads to isotropic domain structures ${ }^{11,12}$, strongly anisotropic domains up to a self-stratified film are expected in the vicinity of any surface confining a bulk sample ${ }^{13,14}$. Interactions with the substrate and the vacuum surfaces can add dewetting as a further structure directing mechanism. Spinodal features normally connected to phase separation are also observed in thin homopolymer films as a result of spinodal dewetting ${ }^{15}$. Similarly, a blend system that initially forms a stratified bilayer structure driven by surface energy differences can be further destabilized by liquid-liquid dewetting. In this case, the destabilization forces do not only act at the liquid-air interface, but also at the liquid-liquid interface, leading to significant interfacial roughening. Liquid-liquid dewetting is theoretically well described ${ }^{16,17,18,19}$, but has only recently been verified experimentally ${ }^{20,21}$. Several driving forces are known, and for films below $100 \mathrm{~nm}$ thickness effective molecular interactions between the film surface and the substrate dominate all the other forces ${ }^{22}$. Such interactions may be of van der Waals, electrostatic or entropic type ${ }^{23}$. Liquid interfaces can be additionally destabilized by solvent evaporation. Local cooling at the surface caused by solvent evaporation drives then convective flows (thermocapillar Maragoni convection) $^{24,25}$.

We here introduce two systems where we create nano-structured interfaces employing the guest-material method, but taking advantage of different interactions. The first system is a polymer/guest-polymer blend system, where the structures form via 2-dimensional spinodal decomposition confined between substrate and vacuum interface. The second system consists of a solution of a blend of a carbocyanine dye and a fullerene derivate. Next to their application in silver halide photography, cyanine dyes have interesting photophysical properties that makes them suitable for photovoltaic applications ${ }^{26}$. During spin coating the solution first forms a bilayer, one or both of the layers then destabilizes during the consecutive solvent evaporation. We discuss and compare the two structure formation mechanisms and emphasize the parameters which are most important to influence the feature size.

\section{EXPERIMENTAL}

MEH-PPV (poly[2-methoxy-5-(2'-ethylhexyloxy)-1,4-phenylene-vinylene], $\mathrm{M}_{\mathrm{n}} \sim$ 47000, Aldrich) was purified by dissolving in THF $\left(1 \mathrm{~g} 100 \mathrm{ml}^{-1}\right)$, followed by filtration, precipitation in a tenfold excess of methanol and drying in a vacuum oven at $65{ }^{\circ} \mathrm{C}$. Stock solutions of MEH-PPV $\left(5 \mathrm{mg} \mathrm{ml}^{-1}\right)$ and polystyrene $\left(10 \mathrm{mg} \mathrm{ml}^{-1}\right.$, PS GPC standards from Fluka with molecular weights of $\mathrm{M}_{\mathrm{w}}=3000,10000$, 70000, 150000 and 230000, referred to as PS3k, PS10k, PS70k, PS150k and PS230k, respectively) were prepared in chlorobenzene and mixtures of chloroform and chlorobenzene. Blended films were prepared by mixing together different quantities of MEH-PPV and PS stock solutions before spin coating. The solutions were spin coated onto Mica, which was coated with a $80 \mathrm{~nm}$ thick layer of poly(ethylene dioxythiophene) doped with polystyrene sulfonic acid (PEDOT:PSS, Bayer). The spin rotation parameters were kept constant (acceleration to 3000 rounds per minute in 6 seconds, constant 3000 rounds per minute for 1.5 minutes). If necessary, the blend solution was diluted to adjust the final average MEH-PPV film thickness to $30-40 \mathrm{~nm}$. PS was removed by immersing the film for 1 minute into the selective solvent cyclohexane.

PCBM ([6,6]-phenyl $\mathrm{C}_{61}$-butyric acid methyl ester) was purchased from Solenne B.V, the Netherlands, 1,1'-diethyl3,3,3',3'-tetramethylcarbocyanine perchlorate (CY) was synthesized in our laboratory following established procedures $^{27}$. PCBM and CY were dissolved in the common solvents chlorobenzene and chloroform. The solutions were 
then spin coated onto glass substrates. The thicknesses were adjusted by varying the concentration and spin speed. The individual parameters as well as the volume fractions of each of the components are specified in the figure captions of the samples of interest. PCBM was removed by immersing the samples in hexane for up to 3 minutes.

Atomic force microscopy (AFM) measurements were performed on a Digital Instruments Multimode Scanning Microscope (Nanoscope III) and on a Nanosurf Mobile S in tapping mode at a resonance frequency of $325 \mathrm{kHz}$ or 170 $\mathrm{kHz}$, respectively. We used rectangular silicon cantilevers (Mikromasch, Nanosensors ${ }^{\mathrm{TM}}$ ) with a typical force constant of $\sim 40 \mathrm{~N} / \mathrm{m}$ and a tip radius of curvature of $\sim 10 \mathrm{~nm}$.

Final average film thicknesses were determined with a Varian Cary 50 UV-VIS spectrophotometer from the dissolved films.

\section{RESULTS}

\subsection{Surface directed spinodal decomposition in a polymer blend}

We first discuss the polymeric blend system of MEH-PPV and PS. We note that spin coated blend films are flat on a 10nanometer scale. In contrast to that, removal of PS with a selective solvent reveals a rough surface, which represents the interface between MEH-PPV and PS which formed during spin coating. This is shown in Figure 1 for different MEHPPV / PS blend films. In mixtures where the guest polymer has a high molecular weight $\left(\mathrm{M}_{\mathrm{w}, \mathrm{PS}}=70 \mathrm{k}, 150 \mathrm{k}\right.$ and $\left.230 \mathrm{k}\right) \mathrm{a}$ pronounced lateral phase separation structure with feature heights larger than $100 \mathrm{~nm}$ dominates the film morphology. Only for the low molecular weight guest polymer mixtures $\left(\mathrm{M}_{\mathrm{w}, \mathrm{PS}}=3 \mathrm{k}\right.$, and $\left.10 \mathrm{k}\right)$, a rather smooth interface is established. During the film formation, two competing processes, bulk-like spinodal decomposition and wetting layer formation, determine the phase morphology. Spinodal decomposition seems to dominate for the high molecular weight guest polymers, while wetting layer formation dominates for the low molecular weight guest polymers.

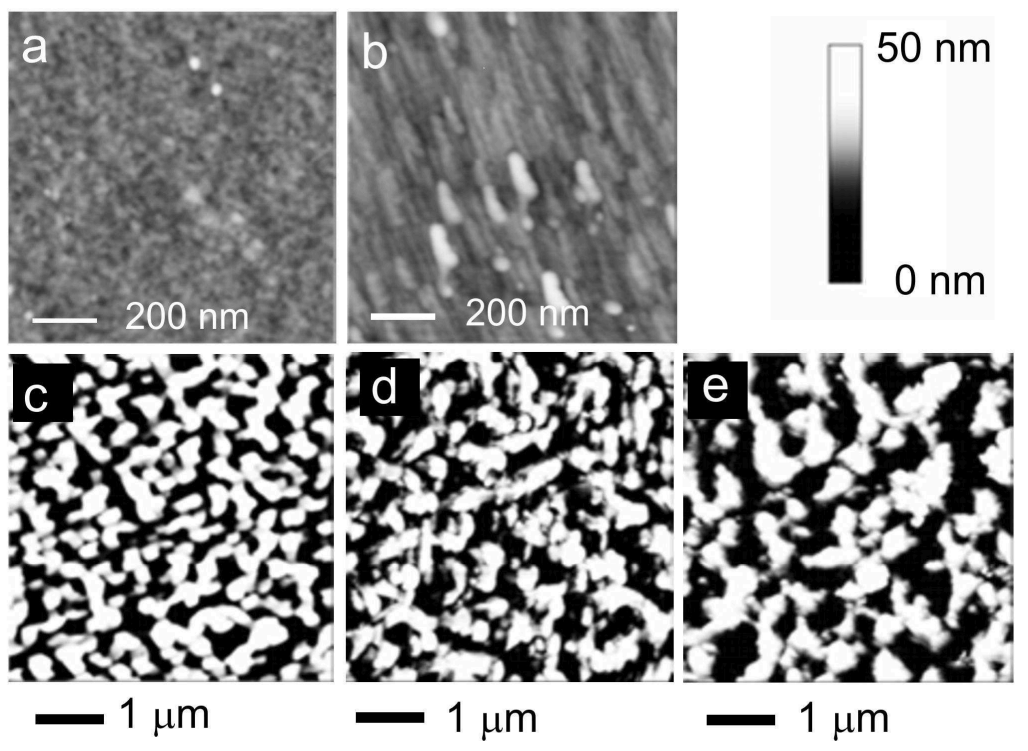

Fig. 1. MEH-PPV/PS film interface imaged with AFM after polystyrene (PS) removal. The films were spin coated from MEH-PPV / PS (1:2 w/w) blends in a 1:1 vol/vol solvent mixture of chlorobenzene and chloroform. The films were prepared from blends with different molecular weights of PS: a) $\mathrm{M}_{\mathrm{w}, \mathrm{PS}}=3000$ (PS3k), b) $\mathrm{M}_{\mathrm{w}, \mathrm{PS}}=10000$ (PS10k), c) $\mathrm{M}_{\mathrm{w}, \mathrm{PS}}=70000$ (PS70k), d) $\mathrm{M}_{\mathrm{w}, \mathrm{PS}}=150000$ (PS150k), and e) $\mathrm{M}_{\mathrm{w}, \mathrm{PS}}=230000$ (PS230k). (Reproduced from reference 10 with permission, Copyright Elsevier 2007). 
To better understand the transition from spinodal- to wetting-directed phase separation, we analyse the structure formation process during spin coating. Spin coating can be understood as a three-step process. In step one, most of the solution is centrifuged off, leaving only a very thin layer on the substrate. In step two, the layer thins further due to solvent evaporation, and in the last step the developed surface structures freeze in. It is inherent to spin coating that many parameters determining the structure formation, like surface and interfacial tensions or viscosities, change continuously.

We qualitatively explain the differences in film formation for samples with different molecular weight with the help of a schematic diagram (Figure 2): Immediately after coating the preferential interactions of MEH-PPV with the PEDOTPPS and of PS with the vacuum surface lead to the formation of enrichment layers of MEH-PPV against the substrate and of PS against the vacuum surface (A in Figure 2). It is only below a smaller solvent concentration that phase separation between the two components starts from the mixture (indicated by point B in Figure 2). The onset of phase separation strongly depends on the molecular weight of the components. The higher molecular weight blends have a higher effective interaction parameter, resulting in "earlier" phase separation. During solvent evaporation the fluid layer thins and the influence of the substrate and the vacuum interface on the phase behavior gets stronger. In our high PS molecular weight blends, phase separation occurs in a rather thick dilute film with little influence of the substrate. The spinodal pattern of this process will be frozen in during the final solvent evaporation and will collapse onto the substrate (Figure $1 \mathrm{c}, \mathrm{d}, \mathrm{e}$ ). For the low molecular blends, phase separation only starts at a lower solvent concentration, that implies that the film is thinner and the influence of the interfaces on the phase separation kinetics is more pronounced. In that case the enrichment layers stabilize an interface that runs parallel to the surfaces (Figure $1 \mathrm{a}, \mathrm{b}$ ).

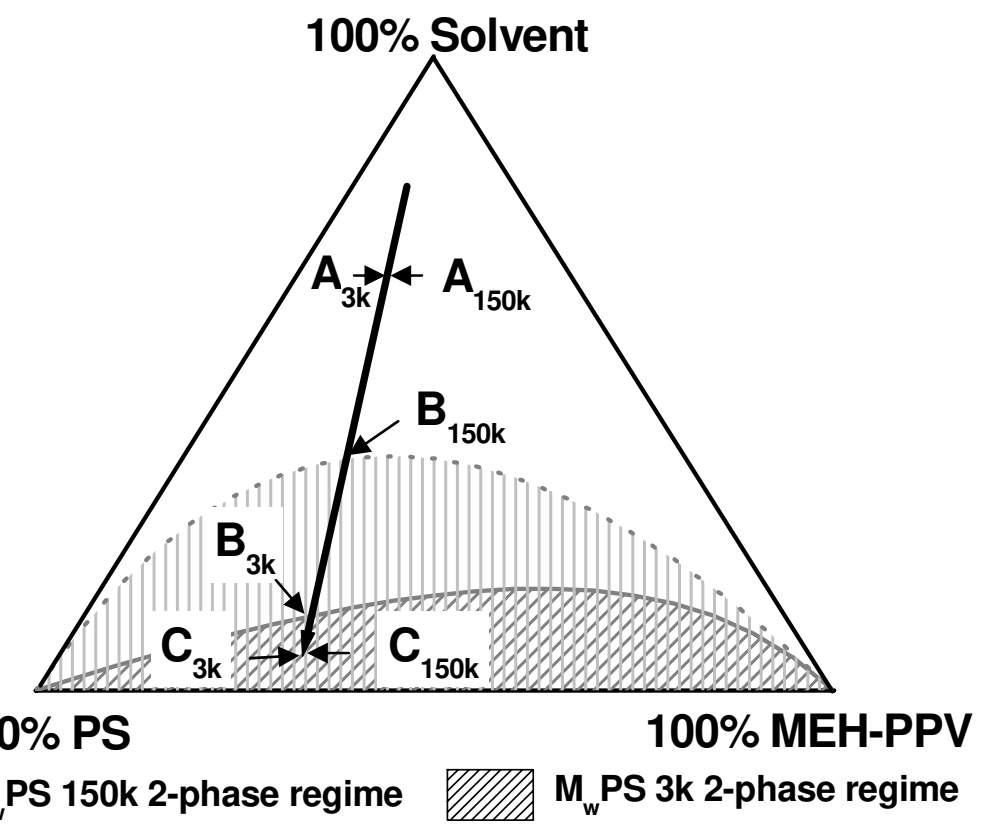

Fig. 2. Schematic ternary phase diagram of a polymer / polymer / solvent system in the bulk for two different polymer molecular weights. The influence of the surfaces on the shape of the spinodal is not taken into account. During the solvent quench the system undergoes numerous transitions: (A) Enrichments layers form at the surfaces, (B) Spinodal decomposition sets in, (C) Glass transition. While the formation of surface enrichment layers and the glass transition is quasi independent of molecular weight, the spinodal line depends strongly on molecular weight. 
The diagram of Figure 2 does not account for the effect of the interface potential on the shape of the bulk phase diagrams. These rather complex interactions will finally induce wetting transitions, capillary condensation or interface localization/delocalizations. These effects may only be proved by resolving the full dynamics of the spin coating process. A more detailed surface scan of the low molecular weight guest polymer mixtures also reveals nanometer sized spinodal patterns at the interface (Figure 3), indicating the influence of additional interactions.

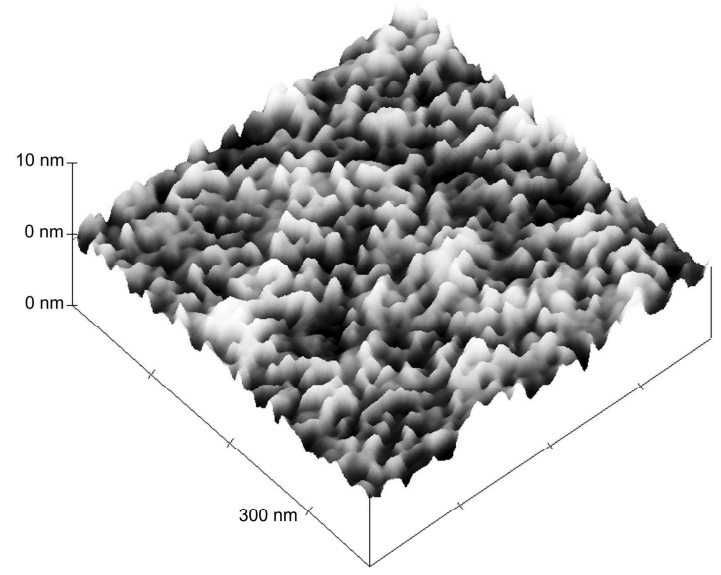

Fig. 3. AFM image of a MEH-PPV surface after removal of the PS phase. The film was spin coated from a MEH-PPV / PS3k $(1: 2 \mathrm{w} / \mathrm{w})$ mixture in chlorobenzene. The Fourier transform of this surface shows a peak at $166 \mathrm{~nm}$ which corresponds to the coarse surface modulations (contrast white-black). This superstructure can be explained by bulk spinodal decomposition in analogy to the decomposition pattern shown in Figs. 1c-e. In addition, a plateau at smaller wavelengths $(\sim 27 \mathrm{~nm})$ corresponds to substructures with dimensions below $10 \mathrm{~nm}$. (Reproduced from reference 10 with permission, Copyright Elsevier 2007).

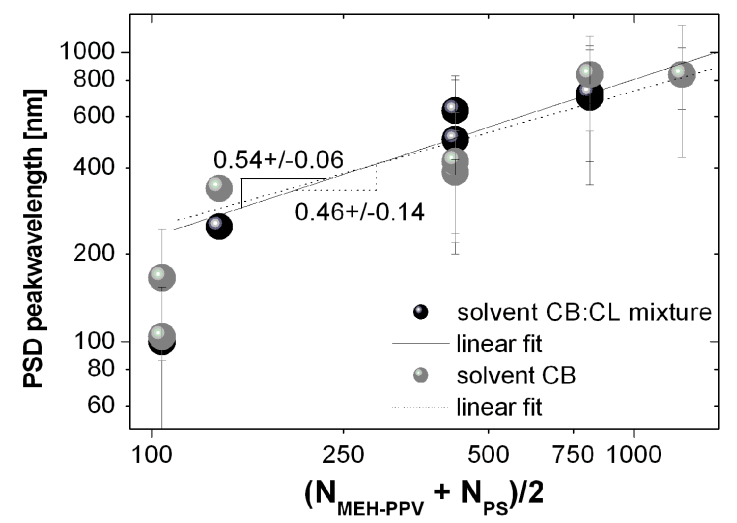

Fig. 4. Double-logarithmic plot of the spinodal wavelengths of MEH-PPV surfaces after removal of PS, determined from the power-spectra vs the mean of the degrees of polymerization. Films were spin coated from 1:2 w/w MEH-PPV / PS blends from a mixture $(1: 1 \mathrm{vol} / \mathrm{vol})$ of chlorobenzene $(\mathrm{CB})$ and chloroform $(\mathrm{CL})$, and of CB only. Linear fits are plotted independently for two different datasets (solvents). The linear fit was performed excluding the two PS3k samples. The error bars indicate the wavelength where the 2D isotropic power spectral density (PSD) peak dropped to one half. 
We executed Fourier transformations on the topographical images obtained by AFM and observed ringlike structures. A plot of the characteristic wavelengths as a function of the arithmetical mean of the degrees of polymerization is shown in Figure 4. We observe a systematic decrease of wavelength with decreasing molecular weight and a marked drop in wavelength for the PS3k sample. Excluding PS3k from the analysis, the data follow a scaling law with critical exponents $\sim 0.54$ and $\sim 0.46$ for samples spin coated from a mixture of chlorobenzene and chloroform, and from chlorobenzene only. The values of the scaling exponents indicate that bulk spinodal decomposition plays the dominant role in the structure formation mechanism, since the molecular weight dependence of the correlation length of concentration fluctuations $(\xi)$ in a bulk sample ${ }^{28,29}$ is given by $\xi \sim\left(\right.$ degree of polymerization $\mathrm{N}$ ) ${ }^{0.5}$ for a symmetrical polymer blend with $\mathrm{N}=\mathrm{N}_{\mathrm{A}}=\mathrm{N}_{\mathrm{B}}$. A different mechanism must be responsible for the additional very fine spinodal patterns observed for low guest polymer molecular weights.

\subsection{Liquid-liquid dewetting in a blend of small molecules}

In the following section, we describe the second system under consideration, a blend solution of PCBM and CY. In Figure 5a we show typical dewetting patterns which are observed in a spin coated blend film. The system decomposes into domains which are embedded in a matrix of the other phase. PCBM is easily identified as the domain-forming component. In Figure 5b we show a corresponding AFM image after removal of PCBM with the selective solvent hexane.
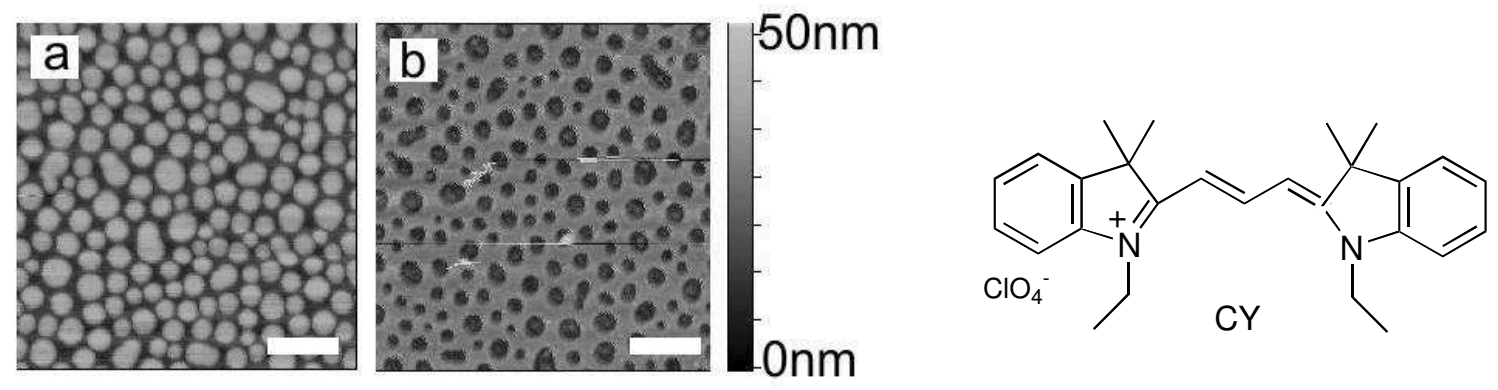

Fig. 5 a) AFM image of a spin coated PCBM/CY film with a molecular weight ratio of PCBM/CY $=0.9$. PCBM forms isolated droplets. 5b) The film shown in 5a) after removal of the PCBM domains with hexane. The PCBM domains fully penetrated the CY matrix. The CY layer is $100 \mathrm{~nm}$ thick. The scale bar is $1 \mu \mathrm{m}$ long. Also shown is the chemical structure of the cyanine dye CY.

From our experimental findings we infer that the mixed solution during spin coating does not phase separate on a microscopic scale, but transforms into a macroscopic bilayer, with a CY film at the substrate interface and a PCBM film at the vacuum interface. Transient bilayer formation during spin coating from a mixed solution is a commonly observed phenomena in polymers ${ }^{30}$ and small molecules ${ }^{21}$. In a next stage the film thins due to solvent evaporation and the toplayer becomes unstable. The exact nature of the instability is difficult to deduce unambiguously (thermo- and solutocapillarity during evaporation may play a role in the destabilization of the film as well), but once the overall film thickness drops below $100 \mathrm{~nm}$, effective molecular interactions between the surfaces become dominant. The dipole in the CY molecule may strongly contribute to van der Waals interactions. We do also not exclude that the mobile $\mathrm{ClO}_{4}{ }^{-}$counterions diffuse into PCBM and the destabilization force develops an electrostatic character. In the last cases, destabilization will only set in in the last stages of film formation where the samples almost reached their final thickness. The destabilization forces amplify thermal or other fluctuations at the film interfaces and initiate spinodal dewetting. A schematic sketch of the envisioned process is depicted in Figure 6. A Fourier analysis of the surface topography indeed shows the ring structure typical for a spinodal process. The initial linear modes will develop large amplitudes until film rupture. Differently from conventional dewetting on a solid substrate, not only the liquid-air interface but also the liquidliquid interface is subject to deformation. The growth of the penetration is kinetically controlled and is influenced by the 
film viscosities ${ }^{31,32}$. As the penetration amplitude increases, the intermolecular forces in the lower layer also increase and eventually lead to rupture in the lower film. The film we show in Figure 5 captures a stage where the upper layer of PCBM has ruptured and fully penetrated the lower layer during final solvent evaporation. However, the parameter space of a bilayer system offers endless distinct final film morphologies.
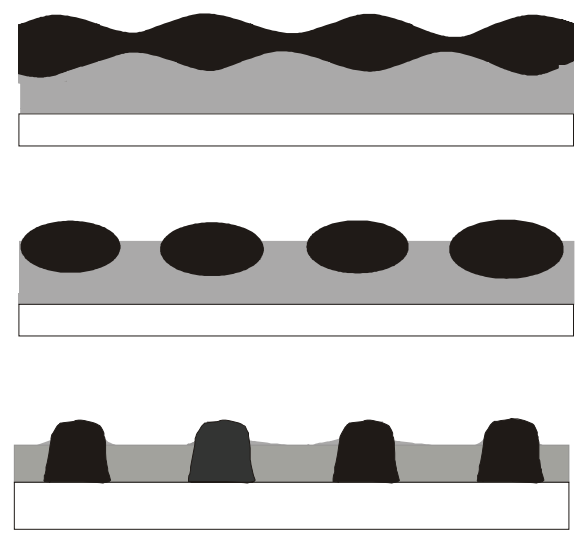

Spinodal wavelength $\lambda$

Fig. 6. Schematic drawing of the envisioned structure formation process: The upper layer dewets and penetrates the lower layer. That will locally decrease the lower layer thickness which favors spinodal break-up of the lower layer. During the final solvent evaporation the PCBM domains sink to the substrate.

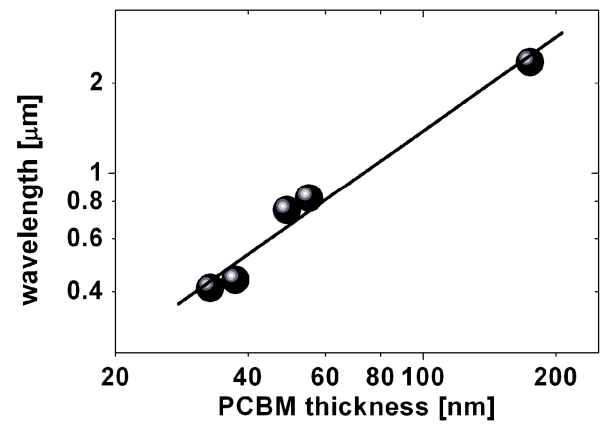

Fig. 7. Film thickness dependence of the spinodal wavelength of the PCBM domains for films with a molecular weight ratio of PCBM to $\mathrm{CY}=1: 1$. The CY film has a thickness similar to PCBM. The dominant spinodal wavelength has been extracted from the fourier transforms of the AFM images.

We can explore the possibilities to further increase the interfacial area. That implies a decrease of the lateral dimensions and an increase of the amplitude of the instability. If we e.g. assume that solely van der Waals forces act between the two parallel interfaces as driving force for film destabilization, the interaction energy varies with film thickness as $\mathrm{A} / \mathrm{d}^{2}$, where A is the Hamaker constant. A positive Hamaker constant for a single layer implies an attractive force leading to spinodal film destabilization, a negative value corresponds to a negative force leading to perfect wetting ${ }^{22}$. In a bilayer film, the interactions of the gas (air) with the substrate across layers 1 and 2, of layer 2 (PCBM) with the substrate across 
layer 1, and of layer 1 (CY) with the gas (air) across layer 2 (PCBM) determine the overall film instability. Different from the spinodal decomposition observed in the MEH-PPV/PS system, the wavelength in spinodal dewetting depends critically on the layer thickness. When the thickness $\Delta$ of the upper layer is small compared to the total film thickness, the dominant wavelength of the instability in the upper layer increases as $\lambda \sim \Delta^{2}{ }^{33}$. Consequently, a thinner upper layer will result in smaller lateral features. We indeed observe a distinct thickness dependence of the lateral features on the final film thickness (Figure 7).

For efficient light absorption in solar cells, the thickness of the CY film should not drop below $30 \mathrm{~nm}$, corresponding to an absorbance of $\sim 0.5$. In a film with a 1:1 molecular weight mixing ratio of PCBM to CY, that will limit the smallest lateral dimensions of the structures we can reach by the primary dewetting process to about $\lambda=400 \mathrm{~nm}$ (according to figure 7). Only a secondary process typical for dewetting helps to reduce the lateral dimensions further: when the PCBM domains penetrate the underlying CY film, the material pushed out of the CY layer accumulates in a rim surrounding the PCBM. This is a process also commonly observed during conventional dewetting ${ }^{34}$. We depict the process in Figure 8.
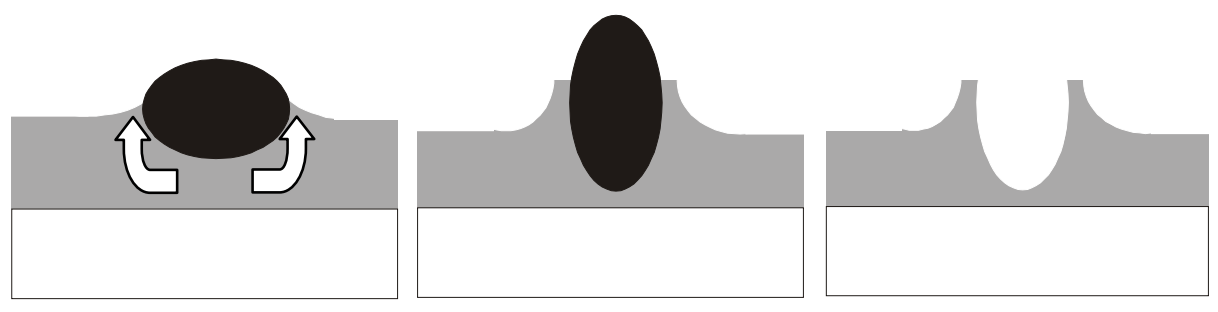

Fig. 8. Schematic drawing of the rim formation process. After rupture of the PCBM film, the PCBM domains (black) penetrate the lower CY layer (grey). A flow of material from the lower layer forms a rim around the PCBM domains. After removal of the PCBM, a CY film with distinct surface features as shown in the AFM images in figure 9 remains.
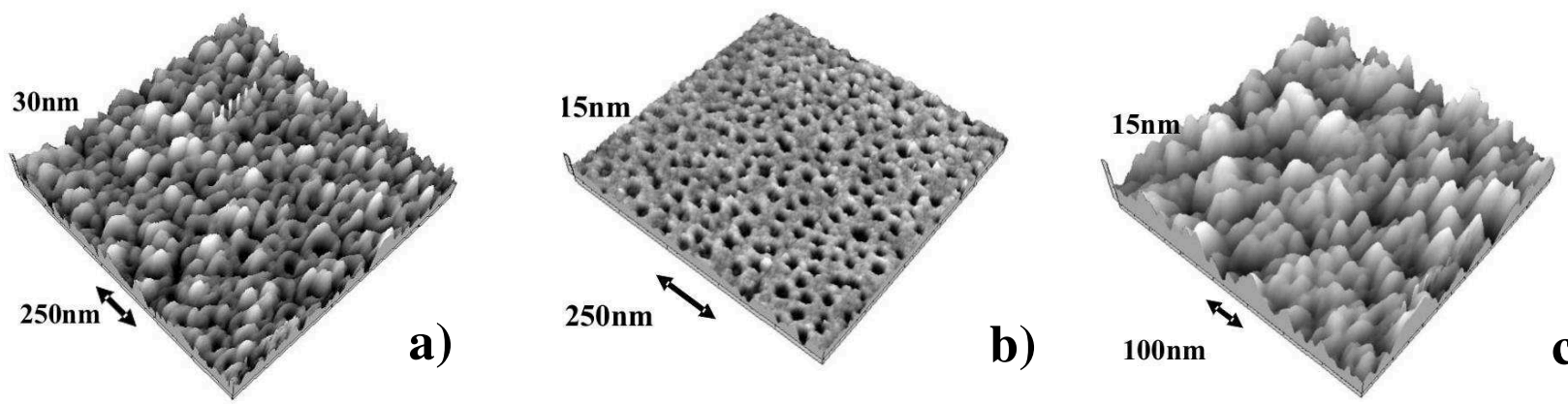

c)

Fig. 9. Rim formation in CY films during hole formation by the penetrating PCBM domains. PCBM is removed in hexane and the surface structure is imaged by AFM. a) Film spin coated from a mixture with a PCBM/CY molecular weight mixing ratio of 0.67 . The average $\mathrm{CY}$ film thickness is $50 \mathrm{~nm}$. b) Film spin coated from a mixture with a PCBM/CY molecular weight mixing ratio of 0.41 . The average CY film thickness is also $50 \mathrm{~nm}$, but the initial PCBM layer was thinner, leading to a smaller spinodal wavelength. Also the amplitude of the rims in the underlying CY film is reduced. c) Ultra thin film spin coated from a mixture with a PCBM/CY molecular weight mixing ratio of 2.0. The average CY film thickness is $5 \mathrm{~nm}$. In all cases, the lower layer did not rupture.

The rim structure is most clearly visible after PCBM removal. Examples are shown in Figure 9. Depending on thickness and volume fraction ratio different morphologies can be realized. The lateral dimensions of the structures depend mainly 
on the initial PCBM thickness. While a thinner PCBM film leads to smaller lateral dimensions, also the amplitude of the features is reduced. The maximum surface roughness is thus found by optimizing the PCBM thickness with respect to lateral and height dimension. In Figure $9 \mathrm{c}$ we show the undulations in the underlying CY film that was covered by an ultra thin (10nm) PCBM layer. The lateral dimensions are well below 50nm.

\section{CONCLUSION}

An initial film morphology of interest for photovoltaic applications needs to show the following features: (i) a donor wetting layer is fully established at the anode interface, and (ii) the lateral length scales of the topographical surface patterns are well below $100 \mathrm{~nm}$. Both of these features could be observed in spin coated blends of MEH-PPV and PS of molecular weight $\mathrm{M}_{\mathrm{w}}=3000$ as well as in spin coated blends of PCBM and CY. The mechanisms that lead to these nanostructures differ in both cases. For the MEH-PPV/PS system, bulk like spinodal decomposition in the solventpolymer-polymer blend is the dominating structure formation mechanism. The observed nanoscale structures only develop when distinct MEH-PPV and PS wetting layers are formed. For the PCBM-cyanine dye system, differences in surface energies of the two components first initiate macro-phase separation of the system into a bilayer during spin coating. The interfacial structures result from dewetting and penetration of the upper layer from and into the bottom layer, respectively. Rims form around the regions where PCBM domains penetrate the lower layer and are a source of significant interfacial roughening. Similiar as in the polymer-polymer system, it is not the primary phase separation process which generates nanometer scale features, but a secondary effect.

Both approaches offer the opportunity to engineer surfaces with desired features. The polymer-polymer interface can be most efficiently controlled by varying the effective polymer-polymer interaction parameter. We demonstrated this by varying the molecular weight, but a comparable effect can be gained by choosing different polymer combinations. Surface interactions strongly depend on the layer thickness, it is thus the film thickness combined with a optimized mixing ratio of the two components which allows us to control the features in the PCBM-cyanine system.

\section{Acknowledgements}

The authors thank T. Geiger (Empa) for the synthesis of CY. We acknowledge funding from COST (Coopération européenne dans le domaine de la recherché scientifique et technique).

\section{REFERENCES}

1. G. A. Chamberlain, "Organic solar cells: A review", Solar Cells 8, 47 (1983).

2. C. J. Brabec, N. S. Sariciftci and J. C. Hummelen, "Plastic solar cells," Adv. Funct. Mat. 11(1), 15-26 (2001).

3. H. Hoppe and N. S. Sariciftci, "Organic solar cells: An overview, " J. Mater. Res. 19(7), 1924-1945 (2004).

4. C. W. Tang, "Two-layer organic photovoltaic cell," Appl. Phys. Lett. 48(2), 183-185 (1986).

5. G. Yu and A. J. Heeger, "Charge separation and photovoltaic conversion in polymer composites with internal donor-acceptor heterojunction, " J. Appl. Phys. 78, 4510-4513 (1995).

6. J. J. M. Halls, C. A. Walsh, N. C. Greenham, E. A. Marseglia, R. H. Friend, S. C. Moratti and A. B. Holmes, "Efficient photodiodes from interpenetrating polymer networks," Nature 376, 498-500 (1995).

7. L. Chen, D. Godovsky, O. Inganäs, J. C. Hummelen, R. A. J. Janssens, M. Svensson and M. R. Andersson, "Polymer photovoltaic devices from stratified multilayers of donor-acceptor blends," Adv. Mater. 12(18), 1367-1370 (2000).

8. M. Drees, K. Premaratne, W. Graupner, J. R. Heflin, R. M. Davis, D. Marciu and M. Miller, "Creation of a gradient polymer-fullerene interface in photovoltaic devices by thermally controlled interdiffusion," Appl. Phys. Lett. 81, 46074609 (2002). 
9. F. A. Castro, H. Benmansour, C. F. O. Graeff, F. Nüesch, E. Tutis and R. Hany, "Nanostructured organic layers via polymer demixing for interface-enhanced photovoltaic cells," Chem. Mater. 18, 5504-5509 (2006).

10. F. A. Castro, C. F. O. Graeff, J. Heier and R. Hany, "Interface morphology snapshots of vertically segregated thin films of semiconducting polymer/polystyrene blends," Polymer 48, 2380-2386 (2007).

11. P. G. De Gennes, "Dynamics of fluctuations and spinodal decomposition in polymer blends," J. Chem. Phys. 72(9), 4756-4763 (1980).

12. A. Budkowski, "Interfacial phenomena in thin polymer films," Adv. Polym. Sci. 148, 1-111 (1999).

13. R. A. L. Jones, L. J. Norton, E. J. Kramer, F. S. Bates and P. Wiltzius, "Surface-directed spinodal decomposition," Phys. Rev. Lett. 66, 1326-1329 (1991).

14. F. Bruder and R. Brenn, "Spinodal decomposition in thin films of a polymer blend," Phys. Rev. Lett. 69(4), 624-628 (1992).

15. R. Seeman, S. Herminghaus, C. Neto, S. Schlagowski, D. Podzimek, R. Konrad, H. Mantz and K. Jacobs, "Dynamics and structure formation in thin polymer melt films," J. Phys.: Condens. Matter 17, S267-S290 (2005).

16. F. Brochard-Wyart, P. Martin and C. Redon, "Liquid/liquid dewetting," Langmuir 9(12), 3682-3690 (1993).

17. A. Pototsky, M. Bestehorn, D. Merkt and U. Thiele, "Alternative pathways of dewetting for a thin liquid two-layer film," Phys. Rev. E 70, 025201 1- 0252014 (2004).

18. A. Pototsky, M.Bestehorn, D. Merkt and U. Thiele, "Morphology changes in the evolution of liquid two-layer films," J. Chem. Phys. 122(22), 224711-224724 (2005).

19. D. Bandyopadhyay, R. Gulabani and A. Sharma, "Instability and dynamics of thin liquid bilayers," Ind. Eng. Chem. Res. 44(5), 1259-1272 (2005).

20. A. M. Higgins and R. A. L. Jones, "Anisotropic spinodal dewetting as a route to self-assembly of patterned surfaces," Nature 404, 476-478 (2000).

21. L. V. Govor, G. Reiter, G. H. Bauer and J. Parisi, "Bilayer formation in thin films of a binary solution," Phys. Lett. A 353, 198-204 (2006).

22. J. N. Israelachvili, Intermolecular and Surface Forces, Academic Press, London, 1992.

23. R. J. Hunter, Foundation of Colloid Science, vol 1, Clarendon Press, Oxford, 1992.

24. L. E. Scriven and C. V. Sternling, "The Marangoni effects," Nature 187, 186-188 (1960).

25. J. R. A. Pearson, "On convection cells induced by surface tension," Fluid Mech. 4(5), 489-500 (1958).

26. F. Meng, K. Cheng, H. Tian, L. Zuppiroli and F. Nueesch, "Cyanine dye acting both as donor and acceptor in heterojunction photovoltaic devices," Appl. Phys. Lett. 82(21), 3788-3790 (2003).

27. L. A. Ernst, R. K. Gupta, R. B. Mujumdar and A. S. Waggoner, "Cyanine dye labeling reagents for sulfhydryl groups," Cytometry 10(1), 3-10 (1989).

28. M. Müller and K. Binder, "Wetting and capillary condensation in symmetric polymer blends: A comparison between Monte Carlo simulations and self-consistent field calculations," Macromolecules 31(23), 8323-8346 (1983).

29. K. Binder, "Collective diffusion, nucleation, and spinodal decomposition in polymer mixtures," J. Chem. Phys. 79(12), 6387-6409 (1983).

30. S. Y. Heriot and R. Jones, "An interfacial instability in a transient wetting layer leads to lateral phase separation in thin spin-cast polymer-blend films," Nat. Mat. 4, 782-786 (2005).

31. P. Lambooy, K. C. Phelan, O. Haugg and G. Krausch, "Dewetting at the liquid-liquid interface," Phys. Rev. Lett. 76(7), 1110-1113 (1996).

32. Q. Pan, I. K. Winey, H. H. Hu and R. J. Composto, "Unstable polymer bilayers. 2. The effect of film thickness," Langmuir 13, 1758-1766 (1997).

33. A. Oron, S. H. Davis and S. G. Bankoff, "Long-scale evolution of thin liquid films," Rev. Mod. Phys. 69(3), 931980 (1997).

34. R. Seemann, S. Herminghaus and K. Jacobs, "Dewetting patterns and molecular forces: A reconciliation," Phys. Rev. Lett. 86(24), 5534-5537 (2001). 